\title{
Implementing opportunistic spectrum access in LTE-advanced
}

\author{
Vicente Osa ${ }^{1 *}$, Carlos Herranz $^{1}$, Jose F Monserrat ${ }^{1}$ and Xavier Gelabert ${ }^{2}$
}

\begin{abstract}
Long term evolution advanced (LTE-A) has emerged as a promising mobile broadband access technology aiming to cope with the increasing traffic demand in wireless networks. However, the enhanced spectral efficiency offered by LTE-A may become futile without a better management of scarce and overcrowded electromagnetic spectrum. In this sense, cognitive radio (CR) has been proposed as a potential solution to the problem of spectrum scarcity. Among all the mechanisms provided by CR, opportunistic spectrum access (OSA) aims at a dynamic and seamless use of certain licensed bands provided the licensee is not harmfully affected. This operation requires spectral awareness in order to avoid interferences with licensed systems. In spite of implementing some spectrum sensing mechanisms, LTE-A technology lacks other tools that are needed in order to improve the knowledge of the radio environment. This work studies the adoption of a Geo-located data base (Geo-DB) that cooperatively retrieves and maintains information regarding the location of unutilized portions of spectrum potentially available for OSA. Moreover, the potential benefit of this LTE-compliant OSA solution is evaluated using a calibrated simulation tool, by which numerical results allow us to optimally configure the system and show that the proposed opportunistic system is able to significantly improve its performance.
\end{abstract}

\section{Introduction}

Long term evolution (LTE) was designed to improve mobile broadband, supporting higher-rate services [1]. However, the road towards IMT-Advanced systems, such as LTE-Advanced (LTE-A), poses more ambitious requirements and thus some challenging new techniques are still being discussed in the framework of 3rd generation partnership project (3GPP) to reach or go beyond these requirements. As one of their key features, all IMT-Advanced technologies foresee the aggregation of continuous or discontinuous portions of spectrum in order to achieve wider bandwidth and consequently increase transmission rate capability [2]. This concept is usually known as Carrier Aggregation (CA). Several studies (see e.g., [3]) reveal an important increase in average user throughput when CA is performed while celledge user throughput remains unaffected. However, despite the potential advantages of $\mathrm{CA}$, a major problem nowadays is the reduced amount of available and useful spectrum for future mobile technologies.

\footnotetext{
* Correspondence: viosgi@iteam.upv.es

'Universitat Politècnica de València, iTEAM Research Institute, Camino de Vera S/N, 46022 València, Spain

Full list of author information is available at the end of the article
}

Profitable electromagnetic spectrum for mobile communication applications is a scarce resource whose use is strictly and rigidly licensed by governmental entities (e.g., FCC in the US, OFCOM in the UK, etc.). Besides, current spectrum utilization is not uniform, that is, some bands are heavily exploited while others remain lightly-used, both in the temporal and spatial domains. Literature refers to these underutilized portions of spectrum as spectrum holes or white spaces [4]. Cognitive radio $(\mathrm{CR})$ has been proposed as a feasible solution to this inefficient use of the radio spectrum $[5,6]$ providing a set of methodologies and functionalities in order to cope with this burden. Among the functionalities provided by $C R$, opportunistic spectrum access (OSA) is devised as a dynamic method to increase the overall spectrum efficiency by allowing non-licensed (a.k.a. cognitive or secondary) users to utilize unused licensed (or primary) spectrum. For this purpose, a correct identification of channel ${ }^{a}$ vacuity conditions by means of spectrum awareness techniques becomes fundamental. Specifically, we must ensure that, (1) a channel targeted for secondary use is actually not being utilized by a licensed user; and (2) the channel occupied by a secondary user is promptly vacated if a primary user suddenly 
appears. Otherwise, OSA might cause a harmful interference with the licensed activity. Considering the abovementioned premises, efficient spectrum awareness enables an opportunistic use of otherwise unutilized idle bands. Noticeably, the Digital TV (DTV) band is a typical example of inefficient spectrum use since, depending on the geographical location, only certain channels are occupied. This fact has been noticed by standardization bodies that are working to make possible spectrum sharing without causing harmful interference to the primary (or licensed) system [7-9]. In [10], the use of free TV channels by wireless technologies was studied showing that there is a clear opportunity to enhance the performance of IMT-Advanced systems by exploiting, for instance, the released frequency band resulting from the analog-to-digital TV switch-over, referred to as digital dividend (DD) in literature [11]. Zhao et al. investigated the framework of spectrum sharing schemes based on cognitive sensing for the LTE-A and DTV coexistence [12]. However, less attention was paid on the practical issues related to the implementation of this framework.

Channel status, the keystone of CR, can be easily acquired using spectrum sensing, which is usually performed by the secondary users that report the spectrum measurements to the system. An important issue in spectrum sensing is the reliability of the partial measurements performed by a single user. It is well known that mobile radio systems suffer from multipath fading and shadowing, causing severe degradation to the signal, which may lead opportunistic secondary users not to detect the primary activity in a certain moment or location [13]. Moreover, sensors may also suffer from the hidden node problem in which the primary signal strength at the non-licensed user position is weak but opportunistic transmission will still interfere with the licensed operation. In order to mitigate these drawbacks, longer observation times are suggested to improve performance. $\mathrm{Xu}$ et al. optimized sensing periods and transmission times in energy-constrained CRs [14]. In this sense, it is worth noting that sensing periods cannot be extended sine die since fast opportunity detection is required in practical CR networks [15]. Moreover, increasing sensing times reduces transmission times with a subsequent reduction in data throughput. Cabric et al. addressed spectrum sensing implementation in detail and provided a wide overview of those problems in [16]. Therein, the abovementioned uncertainty of primary activity detection based on a single-sensor is discussed. As a solution to alleviate this problem, they suggest the use of cooperative spectrum sensing. In the framework of OSA, a system is denoted as cooperative if OSA decisions are based not only on the measurements reported by a single user but also on information from other cognitive users. In contrast, in a non- cooperative scheme, decisions are taken regarding the measurements reported by the specific secondary user demanding access to the primary band. It has been shown that cooperative sensing provides reliable detection if the number of cooperating sensors is large enough [17]. In addition, cooperative sensing also shortens the sensing time of the spectrum while improving the overall sensitivity [18]. Nonetheless, related literature does not substantially clarify the optimum cooperative decision criteria to be followed. A comparison between hard decision (i.e., decision only based on threshold levels) and soft decision-every measurement has a weight in the final decision-is provided in [19], concluding that soft decisions are better-suited for OSA. In the same direction, Xiao et al. suggested a soft cooperative spectrum sensing mechanism based on signal-to-noise ratio (SNR) measurement reports that revealed high performance [20].

Subsequent to spectrum sensing, measurements must be reported to the entity that decides on whether to grant opportunistic access or not. Concerning information exchange for cooperative sensing in CR networks, Pan et al. [21] provided a solution consisting of transmitting low-data control information in a wideband channel avoiding interference with the licensed activity. Similarly, Masri et al. suggested the implementation of a common control channel using ultra wide band (UWB) [22]. However, and to the authors' best knowledge, existing literature lacks feasible and practical implementations of message exchange mechanisms among corresponding entities in a CR network.

Once channel status is known, the geo-localization of these measurements is of great value for a CR network in many ways [23], especially in the case of dynamic OSA. In the existing literature, it is possible to find different schemes that introduce location information for OSA procedures. Specifically, the Federal Communications Commission (FCC) has suggested two well-suited alternatives for a static scenario [24,25]: (1) to check in a centralized database for free resources in the TV band provided the specific cognitive user location is known; or (2) to locally broadcast the availability of free TV channel information. Despite these proposals, the inclusion of location-awareness in a dynamic OSA scenario is a relatively recent research topic which deserves further attention. In [26], a resource allocation scheme is proposed based on the distance between primary and secondary users by predicting the propagation effect and deriving the maximum allocated power. The main disadvantage of this proposal, however, is that spectrum sensing is not incorporated and channel prediction models may lack the required accuracy.

Keeping in mind the above, this article provides specific rules for the practical implementation of a 
cooperative spectrum sensing mechanism in LTE-A. Moreover, this article assesses the suitability of using dynamic OSA in terms of system performance in such networks. It is further proposed the adoption of a geolocated database (Geo-DB) containing the available spectrum holes in a certain frequency band at a given time. In order to maintain and update this database, cognitive users will sense the channel and report measurements using the IEEE 802.21 Multimedia Independent Handover $(\mathrm{MIH})$ standard [27]. The network will retrieve and compile the cooperative sensing measurements and their geographical position in order to decide upon the channel vacuity in a certain location, updating the GeoDB accordingly. This decision should be made considering not only the measurements reported by the cognitive users, but also the confidence in the user's measurement and the distance of the user to the base station, among other factors. Moreover, OSA will be only allowed for those users located inside free primary activity areas. This article also proposes the use of a simple resource allocation scheme combined with a suitable power control mechanism for the opportunistic access to the primary band, based on the Geo-DB information, aiming at minimizing the interference induced to licensed and other secondary users using location as a key element.

The rest of the article is organized as follows. Section 2 describes the tools needed in a LTE-A system to support the Geo-DB containing the location of white spaces. First, different aspects of the spectrum sensing mechanism are analyzed so as to find its optimal set-up. Then, the sensing reporting process and user positioning methods in LTE-A are examined. Subsequently, a possible structure of the Geo-DB is provided. Section 3 describes the OSA mechanism based on a simple, yet efficient, soft cooperative decision algorithm. In addition, this section highlights some constraints that the scheduler must take into account when opportunistically allocating resources in the primary band. The access procedure to those opportunistic resources is also studied, describing some alternatives for signaling purposes. Following, Section 4 shows some relevant results obtained through system-level simulations that highlight the clear benefit that OSA brings to the operation of LTE-A systems. Finally, Section 5 discusses the main conclusions.

\section{Cognitive radio features and tools in LTE- advanced}

This section aims to identify the set of tools available in LTE-A which enables OSA. The objective is to use existing capabilities in order to create a Geo-DB containing the location of white spaces. This analysis focuses on stressing the operational constraints and also on identifying the variables that can be adjusted. Numerical results, presented in Section 4, will provide a reference value for these variables.

\subsection{Potential deployment scenario}

Before going in detail into the OSA enabling mechanisms, it becomes essential to assess the potential scenario where OSA is suitable. In such scenario, an LTE deployment is likely to coexist with other licensed wireless systems implementing different technologies (e.g., DTV, GSM, UMTS, etc.). Some of these licensed systems may not be transmitting persistently, thus underutilizing their legacy frequency bands (e.g., terrestrial trunked radio (TETRA) is mainly used in emergencies); other systems may not use the entire allocated spectrum band (e.g., DTV white spaces). We devise an LTE system to be aware of these spectrum opportunities and to exploit them as long as the opportunity remains, i.e., ceasing transmission once the licensed system starts its activity. As stated in Section 1, among all the available spectrum awareness techniques, we adopt spectrum sensing. Channel status information from each location in the scenario is provided by UEs sensing the spectrum. In order to prevent erroneous channel state declaration, a collaborative spectrum sensing scheme is devised, in which UEs reports their perception of the licensed channel and a final decision about the channel state is made. This information in conjunction with UEs location is used to create the Geo-DB.

In the following sections, the abovementioned set of tools needed to perform our suggested OSA solution are discussed. First, this section discusses spectrum sensing and reporting mechanisms. Then, user location procedure in LTE-A is described. Finally, a possible implementation for the Geo-DB is provided. A further and detailed description of the simulation scenario will be provided in Section 4.1.

\subsection{Spectrum sensing}

The success of OSA depends on the correct channel state detection. Cognitive users must only transmit when the licensed channel is idle so as to not interfere with the primary system. With the aim of avoiding interferences and maximizing secondary transmission opportunities, this section analyzes the spectrum sensing capabilities of LTE-A.

In general, the spectrum sensing task is characterized by the sensing time $\left(T_{s}\right)$ and the sensing period $\left(T_{p}\right)$. Sensing time refers to the time spent to determine the signal strength for a certain frequency band whereas the sensing period determines how often a particular band is monitored by the cognitive user. Some of the user measurement capabilities considered by the LTE-A standard for handover purposes can be exploited to sense 


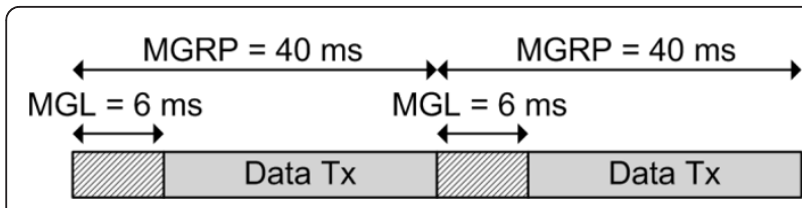

Figure $1 \mathrm{Gap}$ pattern parameters for spectrum sensing in LTE-A.

the primary channel state. Noticeably, the sensing time and sensing period can be directly associated with the gap pattern parameters defined in the standard for UE measurement procedures in the RRC_CONNECTED state [28]: measurement gap length (MGL) and measurement gap repetition period (MGRP). Both parameters are represented in Figure 1.

During sensing (i.e., the gap time period) the scheduler does not allocate resources to the user, which can tune its receiver on other carrier frequencies. According to the standard, MGL is fixed while MGRP is configurable in multiples of the frame length (i.e., $10 \mathrm{~ms}$ ) allowing freedom of choice in the trade-off between up-todate sensing data and system performance. The configuration of MGRP and the set of frequencies to monitor can be done through Radio Resource Control (RRC) signaling, which also guarantees the synchronization between scheduling at the eNodeB (i.e., base station) and sensing at the UE.

The choice of the MGRP should not be made lightly since the system performance heavily depends on this parameter. In detail, the greater this periodicity is, the lesser frequent the channel status is acquired and the more likely the information in the Geo-DB may be outdated. As a result, the probability to allocate an occupied primary resource block $\left(R B^{b}\right)$ to secondary users, that is, the interference or collision probability, is increased. In contrast, higher periodicity means more overhead, consequently reducing data throughput. The effects of changing this parameter will be studied in the Section 4, providing an optimum value for the considered evaluation scenario.

Moreover, LTE-A UEs are capable of measuring the so-called Received Signal Strength Indicator (RSSI). This measurement allows detecting activity/inactivity in the primary band during the measurement gap. Taking into account the re-tuning time required at the beginning and at the end of the measurement gap, it is possible to extract samples in a certain bandwidth during an effective period of $5.166 \mathrm{~ms}$ [[29], pp. 313-314]. In the sensing approach proposed in this article, we consider that in each measurement gap the UE senses one RB (i.e., a bandwidth chunk of $180 \mathrm{kHz}^{\mathrm{c}}$ ) in the licensed band. For example, assuming a hypothetic primary band of 10 $\mathrm{MHz}-50 \mathrm{RBs}-$ and a MGRP of $40 \mathrm{~ms}$, the whole band will be sensed every 2 s. All these measurements must be

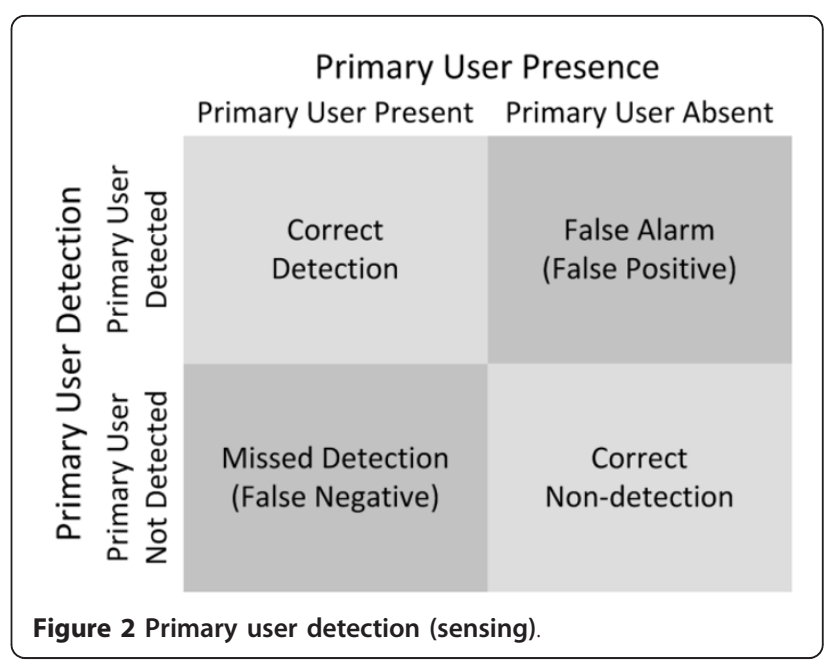

reported to a logical entity that manages and updates the Geo-DB. A proposal for this reporting procedure is provided in Section 3.

In general, the inevitable spectrum sensing inaccuracy may result in erroneous channel occupancy information. Figure 2 shows all possible outcomes that the spectrum sensing mechanism can provide given the presence (i.e., activity) of a primary user in its licensed frequency band. In general, the detection errors-dark-gray areas-are classified into two groups: false positive error or false alarm (FA) and false negative errors or missed detection (MD). FA happens when the presence of an inexistent primary user is detected whereas MD occurs when the cognitive user is unable to detect the primary activity.

The consequences of FA and MD errors are different. A MD error will assign RBs occupied by a licensee to a secondary user, with the consequent interference-or collision-between primary and secondary systems. Conversely, a FA error will lead to spectrum underutilization. In this article, we will model detector performance using the receiver operating characteristic (ROC) curves for the energy detector as calculated in [30,31]. Models for ROC curves represent the FA probability $(\varepsilon)$ as a function of the MD probability $(\delta)$ given some specific channel conditions and design parameters. Specifically, expressions for FA and MD probabilities can be found in the literature under AWGN and fading (Rayleigh, Nakagami, and Ricean) channels. In this study, the Rayleigh channel assumption is considered for which, according to [30], the FA probability for the energy detector is given by

$$
\varepsilon(\lambda)=\frac{\Gamma(m, \lambda / 2)}{\Gamma(m)},
$$

where $\lambda$ is the energy detection threshold, and where $\Gamma(\cdot)$ and $\Gamma(\cdot, \cdot)$ are the gamma and gamma-incomplete 
functions respectively. Parameter $m$ is the so-called time-bandwidth product and relates to the time devoted to sense a particular bandwidth. In general, the larger $m$ is the better our measurements will be. In our specific case, $m$ is fully determined by the time devoted to sense an RB (5.166 ms) and the bandwidth of an RB (180 $\mathrm{kHz})$. Hence, $m=930$.

Similarly, [30] gives the MD probability as

$$
\begin{aligned}
\delta(\lambda)=e^{-\frac{\lambda}{2}} & \sum_{k=0}^{m-2} \frac{1}{k !}\left(\frac{\lambda}{2}\right)^{k}+\left(\frac{1+\bar{\gamma}_{p}}{\bar{\gamma}_{p}}\right)^{m-1} \times \\
& {\left[e^{-\frac{\lambda}{2\left(1+\bar{\gamma}_{p}\right)}}-e^{-\frac{\lambda}{2}} \sum_{k=0}^{m-2} \frac{1}{k !} \frac{\lambda \overline{\gamma_{p}}}{2(1+\bar{\gamma})_{p}}\right], }
\end{aligned}
$$

where $\bar{\gamma}_{p}$ is the received primary SNR which will be considered as an input parameter.

By observing (1) and (2), we note that decision threshold $\lambda$ can be selected for finding an optimum balance between FA probability and the MD probability. However, in practice, see e.g., [32], the threshold is chosen to obtain a certain MD probability rate.

\subsection{Sensing data reporting}

The entire spectrum sensing information obtained by the secondary users must be transmitted to the network for further processing. However, the reporting procedure required for the OSA operation is not specified. One solution could be to use a proprietary communication protocol but this will reduce the viability of OSA in LTE-A. Conversely, we propose the use of the IEEE 802.21 protocol given its popularity and the availability of open source implementations. The IEEE 802.21, a.k.a. Media-Independent Handover (MIH), standard specifies an application-layer protocol aimed to provide soft handover between different 802.xx architectures [27]. Mainly, MIH is based on the exchange of messages reporting a subset of PHY layer events. The MIH functions are enabled by an entity called MIH function (MIHF), which provides MIH event services (MIES), MIH command services (MICS), and MIH information services (MIIS). In this study, only the MIES are of particular interest, in which a local MIHF receives event notifications from a set of well-configured remote MIHFs.

We introduce a new node in the LTE-A network referred to as the cognitive resource manager (CRM), which coordinates the OSA to the unlicensed spectrum based on the notifications reporting the channel status perceived by the cognitive UEs. Based on the above, the CRM must include a MIHF entity which manages the event notification subscription and also receives and processes all notifications concerning spectrum sensing in order to build the Geo-DB. Since the CRM does not perform any sensing task and only compiles MIH event notifications from remote entities, it is necessary to implement a remote MIHF in every LTE-A UE. With the aim of keeping the Geo-DB updated, the CRM needs to know when a cognitive user measures that the RSSI in the licensed band crosses a specific power level or threshold. Therefore, after the attachment procedure, the CRM must send to the active user a MIH_Event_Subscribe message with the list of RBs to be monitored. Moreover, with the MIH_Link_Configure_Thresholds primitive the CRM specifies the thresholds associated with this list.

As explained at the beginning of Section 2.1, using RRC signaling the network can ask LTE-A users to periodically sense the spectrum. Therefore, via the appropriate service access point, the MIHF of the UE can access the measured signal levels and generate notifications according to the sensing information. When the licensed power level crosses the defined threshold, an MIH_Link_Parameters_Report.indication is forwarded to the MIHF in the CRM, which will finally notify it to the upper layers via the MIH user interface. The MIH user specifies an interface between the MIHF and the application that runs the CRM [33]. The MIH user directly sends commands to its local MIHF using the service primitives available in the 802.21 standard, which support event subscription and event notification mechanisms. Figure 3 depicts this data flow among $\mathrm{MIH}$ interfaces from the cognitive UE which perceives a modification in the resource occupancy status and sends the corresponding event notification to the CRM.

The notification event exchange among cognitive users and the CRM is granted using the Default EPS Bearer allocated to any active user in LTE-A. The Default EPS Bearer assigns a unique IP (Internet protocol) address to LTE-A users and provides connectivity, at least, to any node inside the LTE-A network.

\subsection{User positioning}

In the OSA framework, the main objective of user positioning is the collection of dynamic geo-located information of white spaces in the licensed band. This information will help the scheduler to make an opportunistic allocation of resources that could even work when primary activity is detected in distant areas. Section 3 deals with this OSA procedure in more detail.

LTE-A specification considers UE localization through the LTE positioning protocol (LPP) and LPP annex (LPPa) [34-36]. Several different positioning methods are mentioned in the standard, namely: observed time difference of arrival (OTDoA), assisted-Global navigation Satellite system (A-GNSS) and enhanced-cell ID (ECID). Implementation details are omitted here but the interested reader can refer to the standard for further information. All of these positioning methods are based 


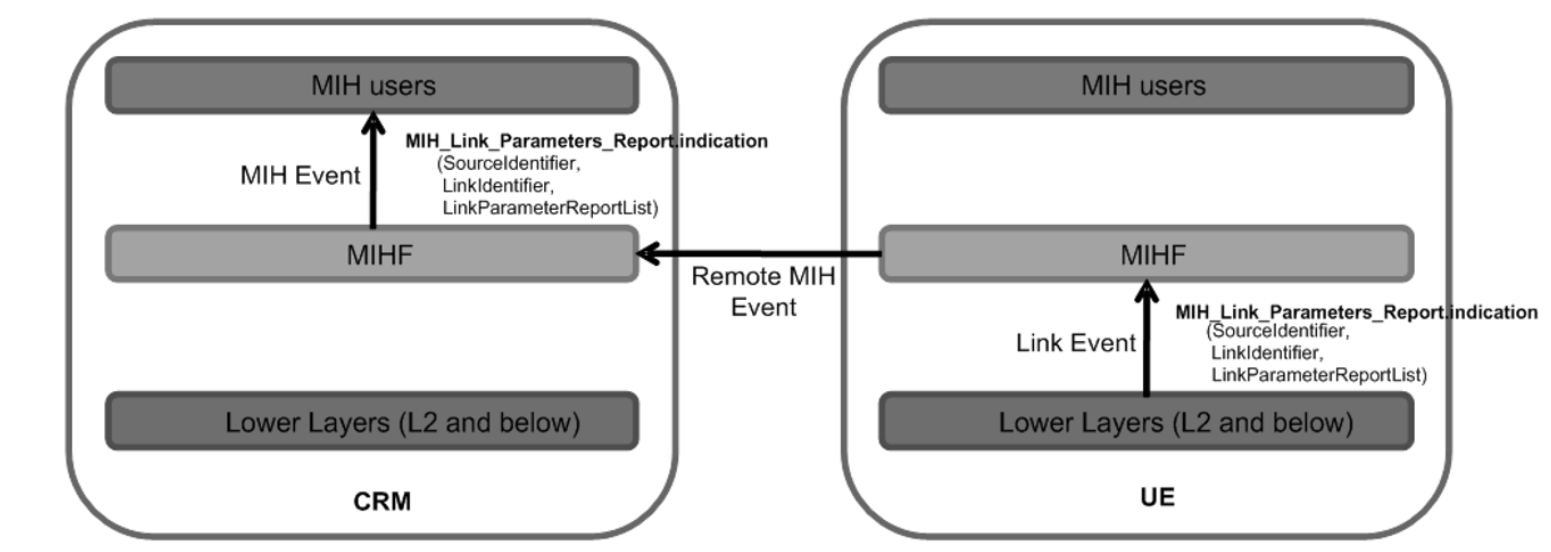

Figure 3 Remote MIH_Link_Parameters_Report.indication event flow.

on measurements collected by the UE or the eNodeB. The mobility management entity (MME) is the entity that receives the request for the localization of a UE from another entity such as another UE, eNodeB or other nodes. Then, the MME sends a location service request to the enhanced serving mobile location centre (E-SMLC), which will execute the positioning procedure through LPP and LPPa protocols. The SLs interface defined between E-SMLC and MME serves as a tunnel for the E-SMLC to transparently carry LPP and LPPa protocols through the MME, in addition to transport the Location Services Application Protocol (LCS-AP) messages and parameters.

The E-SMLC acts as a location server that computes user position using the measurements provided by one or several of the positioning methods. This entity interacts with the UE (using LPP) or the eNodeB (using $\mathrm{LPPa}$ ) through the MME to obtain these measurements. For example, in the case of the E-CID method, the possible measurements, collected by the E-SMLC, are: evolved cell global identifier (ECGI)/physical cell ID, reference signal received power (RSRP), reference signal received quality (RSRQ), UE $R_{x}-T_{x}$ time difference, timing advance (TA) and angle of arrival (AoA). In addition to the collection of this information, the E-SMLC can provide assistance data in the particular cases of AGNSS and OTDoA methods. It is also specified the usage of positioning reference signals (PRS) for OTDoA positioning purposes.

If the UE lacks of A-GNSS functionality, the E-SMLC might combine information from the serving and the neighboring cells in order to triangulate the user's geographical position. Both RSRP and TA allow estimating the distance from the UE to the serving cell, whereas distance to neighboring cells can be derived just from the RSRP values. AoA measures, if available, add more precision to the triangulation process. Table 1 shows the reporting granularity of the measurements involved in the positioning methods [27], which directly affect the positioning precision. It is worth noting that, in the case of TA and UE $R_{x}-T_{x}$ time difference measurements, the resolution can be as good as twice the sampling period, i.e., $2 T_{\text {samp }}$, which corresponds to a spatial resolution of around $10 \mathrm{~m}$.

In the proposed scheme, the CRM and E-SMLC are interconnected using the MME, as shown in Figure 4. The CRM is the entity that requests the location service to the MME, which will activate the E-SMLC service. The resulting location calculated by the E-SMLC is sent to the MME that finally forwards it to the CRM. With the obtained information, the CRM can map sensing reports and location to build the Geo-DB.

\subsection{Geo-located database}

Owing to users' positioning capability, the Geo-DB will contain valuable information about which frequency bands can be used by a given eNodeB at a specific moment of time and the maximum coverage range in order not to interfere with the primary system. Figure 5 illustrates the process of calculating the Geo-DB. The CRM collects the sensing information from the UEs and the positioning information from the location service provided by the MME and updates the database after

Table 1 Measurements reporting granularity

\begin{tabular}{ll}
\hline Measurements & Granularity \\
\hline ECGI/physical cell ID & N.A. (not applicable) \\
RSRP & $1 \mathrm{~dB}$ \\
RSRQ & $0.5 \mathrm{~dB}$ \\
UE R R $^{-} T_{x}$ time difference & If value $<4096 T_{\text {samp }}$ then $2 T_{\text {samp. }}$ \\
TA R $R_{x}-T_{x}$ time difference (TA) & Otherwise, $8 T_{\text {samp }}$ \\
AoA & $2 T_{\text {samp }}$ \\
\hline
\end{tabular}




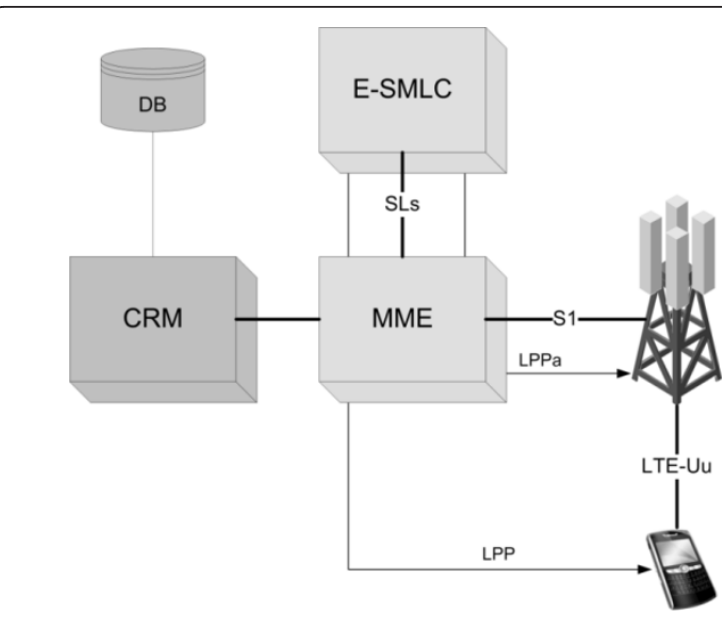

Figure 4 Scheme of the nodes implicated in the Geo-DB maintenance.

the cooperative decision has been taken, which will be explained in Section 3.1. Once this process is finished, the CRM possesses the location of every opportunistic UE and which RBs are suitable for OSA (light-gray RBs in Figure 5) and which not (dark-gray RBs in Figure 5).

The Geo-DB will contain information about the occupation of the different RBs in the licensed spectrum band on a per-cell basis, indicating also the maximum coverage distance from the eNodeB, as shown in Table 2. This way, an eNodeB, identified in the table by its Cell-ID, is able to opportunistically use those RBs with reduced transmission power in case the maximum range is detailed in the corresponding register. Otherwise, the maximum range field is flagged as N.A. and the corresponding resource can be used without restrictions regarding the transmission power. The maximum range

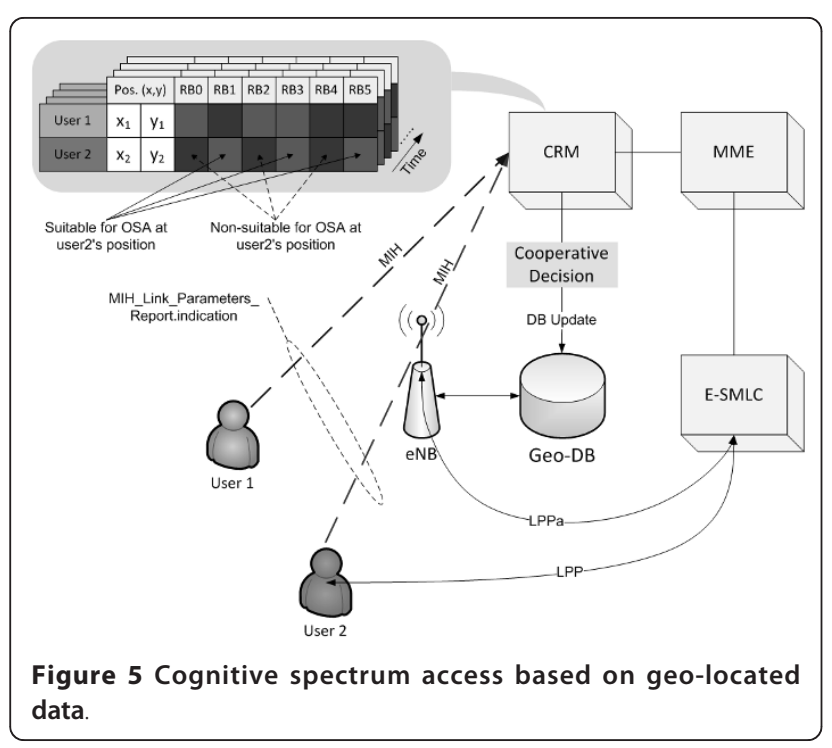

field is expressed in terms of Dist_TA (i.e., timing advance resolution distance), which defines $10 \mathrm{~m}$ width ranges, and represents the maximum distance between the eNodeB and any candidate user. In addition to the data provided in Table 2, the Geo-DB also contains the final decision concerning the different resources as detailed later in Section 3.1.

The information contained in the Geo-DB must be periodically updated in order to take into account the potential utilization patterns in the licensed spectrum, especially if those changes are due to the primary system activity. As stated in Section 2.1, it takes $2 \mathrm{~s}$ for a user to sense a bandwidth of $10 \mathrm{MHz}$. A cooperative decision taking into account the information provided by all the users inside a certain range would allow increasing the sensing accuracy. An up-to-date database will reduce the collision probability that could be caused by the lack of synchronization between the real state of the primary spectrum and the available information stored in the database.

\section{Opportunistic spectrum access procedure}

The proposed OSA procedure relies on the CR tools described in Section 2 and involves executing the processes detailed in this section. Specifically, the cooperative decision on primary system activity in the different monitored channels and the way in which free primary resources are allocated to the users will be addressed. Furthermore, the adopted methodology for the transmission of control information sent to the allocated users informing about the opportunistic resources will conclude this section.

\subsection{Cooperative decision making}

Influenced by mobile channel factors such as noise, shadowing and multipath fading, single sensor measurements are prone to errors. In order to overcome this problem, cooperative spectrum sensing among multiple nodes in different locations is suggested. As mentioned in Subsection 2.3, we propose the implementation of a centralized cooperative spectrum sensing mechanism in order to improve the channel status awareness.

Table 2 An example of some of the data available in the Geo-DB

\begin{tabular}{ccc}
\hline Cell-ID & RB & Max. Range \\
\hline$\ldots$ & & \\
$10001 \mathrm{AX}$ & 0 & 10.Dist_TA \\
$10001 \mathrm{AX}$ & 1 & 12.Dist_TA \\
$\ldots$ & & \\
$10002 \mathrm{AX}$ & 0 & N.A. \\
$10002 \mathrm{AX}$ & 1 & 9.Dist_TA \\
$\ldots$ & & \\
\hline
\end{tabular}


The cooperative decision making mechanism will be implemented in the CRM. For a more efficient use of the available spectrum and to fully exploit the opportunistic nature of our scheme, we consider monitoring and decision-making on a per-RB basis instead of on a per-frequency-band basis (i.e., containing several RBs). The input data considered by the decision mechanism includes all the channel state reports from the UEs served by the same eNodeB and the geographical position of the UEs obtained through the aforementioned location services. Every channel state notification creates a new entry in the CRM containing the UE identifier, the estimated geographical location of the UE, the channel or resource monitored, the licensed activity state sensed in that resource and the time in seconds when the channel state report was received. Once all this data is collected from different UEs, the CRM can make decisions about the vacuity of the monitored resources at different locations. The collected sensing reports are classified by their distance to the eNodeB in different ranges, whose width is given by a multiple of the TA resolution (Dist_TA $=10 \mathrm{~m}$ ). For each range and $\mathrm{RB}$, an independent cooperative decision will be made.

Multiple samples of the sensing data from different resources and UEs are combined in order to update the Geo-DB. For the same UE and sensed resource, only the most recently collected data is used in the cooperative decision calculation (in this study, only measurement reports received no further than $2 \mathrm{~s}$ before the decision is made are considered). The preprocessing carried out by the CRM to update the database is briefly described in Table 3 .

Two simple measure fusion techniques or rules for cooperative decision making are considered in [19]. Therein, the OR-rule states the channel as not free if at least a single UE senses the primary activity. Conversely, the AND-rule decides that the considered resource is occupied if all the reports sense that such RB is occupied.

Inspired by [19], in this study two similar hard-decision rules are introduced. The conservative rule declares the resource as free from licensed activity if all the UEs report such state; otherwise the channel is considered to be occupied. On the other hand, the aggressive strategy declares the resource as idle provided a single UE senses the channel as free. However, due to the

\section{Table 3 Geo-DB update algorithm}

\begin{tabular}{l} 
Geo-DB update algorithm \\
\hline For each cell: \\
For each TA range: \\
Select measurements of UEs inside the TA range \\
Calculate cooperative decision \\
Insert the resulting data into the Geo-DB
\end{tabular}

abovementioned single sensor measurement uncertainty, the different measurements reported over time must be considered in the final decision. That is to say, old measurements must not have the same importance in the final decision as the newest reports due to the fast-changing radio-channel state conditions. Soft-based cooperative decision stands on this idea and, in addition to the abovementioned hard-decision rules, will be also considered in our study.

In soft-based cooperative decision, each reported measure has an associated weight. Following the weighted cooperative spectrum sensing described in [20], instead of weighting the measured primary SNR, we propose to weight the event notifications according to the time when the measurements were triggered and the coherence of the measurements taken inside the same TA range. The CRM will combine the information of the resource state with the weights and will make a decision by comparing the result with a defined threshold. Each resource state notification is weighted according to the elapsed time between the moment the notification was received by the CRM (i.e., the $i$ th notification is received at time $t_{i}$ ) and the instant when the channel state decision is made $-t_{\text {now }}$-as seen in Equation (3). $T_{\text {MAX }}$ is the time elapsed between two consecutive measures of a specific primary resource, that is, $T_{p}$ times the number of primary resources to sense. In addition to this linear weight equation, we will also analyze in Section 4 the quadratic (Equation (4)) and the square root (Equation (5)) version of the formula in order to optimize the performance of the decision algorithm.

$$
\begin{aligned}
& \omega_{i}=\frac{T_{\mathrm{MAX}}-\left(t_{\mathrm{now}}-t_{i}\right)}{T_{\mathrm{MAX}}} \\
& \omega_{i}=\left(\frac{T_{\mathrm{MAX}}-\left(t_{\mathrm{now}}-t_{i}\right)}{T_{\mathrm{MAX}}}\right)^{2} \\
& \omega_{i}=\sqrt{\frac{T_{\mathrm{MAX}}-\left(t_{\mathrm{now}}-t_{i}\right)}{T_{\mathrm{MAX}}}}
\end{aligned}
$$

The decision regarding the resource availability for opportunistic access will be taken according to the value of the spectrum decision metric on a given $\mathrm{RB}$, defined as:

$$
U=\sum_{i=0}^{N-1} \frac{(-1)^{d_{i}} \cdot N_{d_{i}} \cdot \omega_{i}}{N^{2}}
$$

where $d_{i}$ is the state of the monitored resource reported in the $i$ th notification expressed as:

$$
d_{i}= \begin{cases}0 & \text { if resource is sensed as idle } \\ 1 & \text { if resource is sensed as occupied }\end{cases}
$$


$N$ is the number of notification events considered in the decision making mechanism, including free resource notifications, $N_{0}$, and occupied resource notifications, $N_{1} \cdot N_{d_{1}}$ is the number of measurements that agree with the state of the resource notified in the $i$-th measurement report: $N_{0}$ if the resource was sensed occupied, $N_{1}$ otherwise.

As reflected by (6), the spectrum decision metric $U$ will depend on the number of measurements taken into consideration, the weight of each measurement and the most reported single-sensor measurement. If most of the measurements agree on the resource vacuity, a positive value of $U$ is obtained. On the contrary, if the majority of measurements reports that the resource was occupied, a negative value of $U$ is obtained. Note that, in order to normalize the equation, it is needed to divide it by the second power of $N$, in such a way that $-1 \leq U$ $\leq 1$.

Depending on the value of $U$, the considered resource is stated as a candidate for OSA $\left(H_{0}\right)$ if that result is greater than a certain threshold, denoted as $\gamma$. Otherwise, the resource is not available for opportunistic usage $\left(H_{1}\right)$. The decision threshold $\gamma$ must be tuned in order to provide the largest OSA probability without exceeding the interfering limit.

$$
\text { Decision }= \begin{cases}H_{0} & \text { if } U \geq \gamma \\ H_{1} & \text { if } U<\gamma\end{cases}
$$

\subsection{Resource allocation for interference minimization}

As already mentioned, one of the main concerns in CR is the interference minimization towards the licensed system. To this respect, OSA in the licensed band will be only possible inside areas where the mentioned channel is assumed idle. With this aim, the amount of power transmitted in these resources must be dynamically controlled by the non-licensed system. This philosophy is generally known in literature as power control (PC) [37].

Once the Geo-DB is updated and the opportunistic availability of the licensed system resources is confirmed, it is possible to assign these free frequencies-in the form of free RBs-to increment the existing resources available in the LTE system. The dynamic and unpredictable behavior of the licensed channel vacuity forces to set an irregular transmit power profile for the opportunistic resources allocated to each user depending on its distance from the licensed system-power must be low enough so as not to interfere with the primary system. It may happen that no licensed activity is detected in the area served by a given eNodeB. In that case, the LTE system can opportunistically use the available licensed resources in the whole coverage area without power restrictions. On the contrary, if one or several UEs detect the primary activity at any position under the eNodeB coverage area, transmit power must be limited, trying to minimize the interference caused to the primary system. That minimum prohibited distance defines the radius of a circumference inside which OSA is available. Figure 6 explains this concept by showing a simple scenario where a primary system base station and an OSA-capable eNodeB are represented. In this example, the primary and the LTE coverage areasdashed-line ellipses-are partially overlapped, being OSA feasible-solid-line ellipse-in 2.Dist_TA meters away from the eNodeB and the LTE signal in the opportunistic band must not exceed the limits of the OSA area.

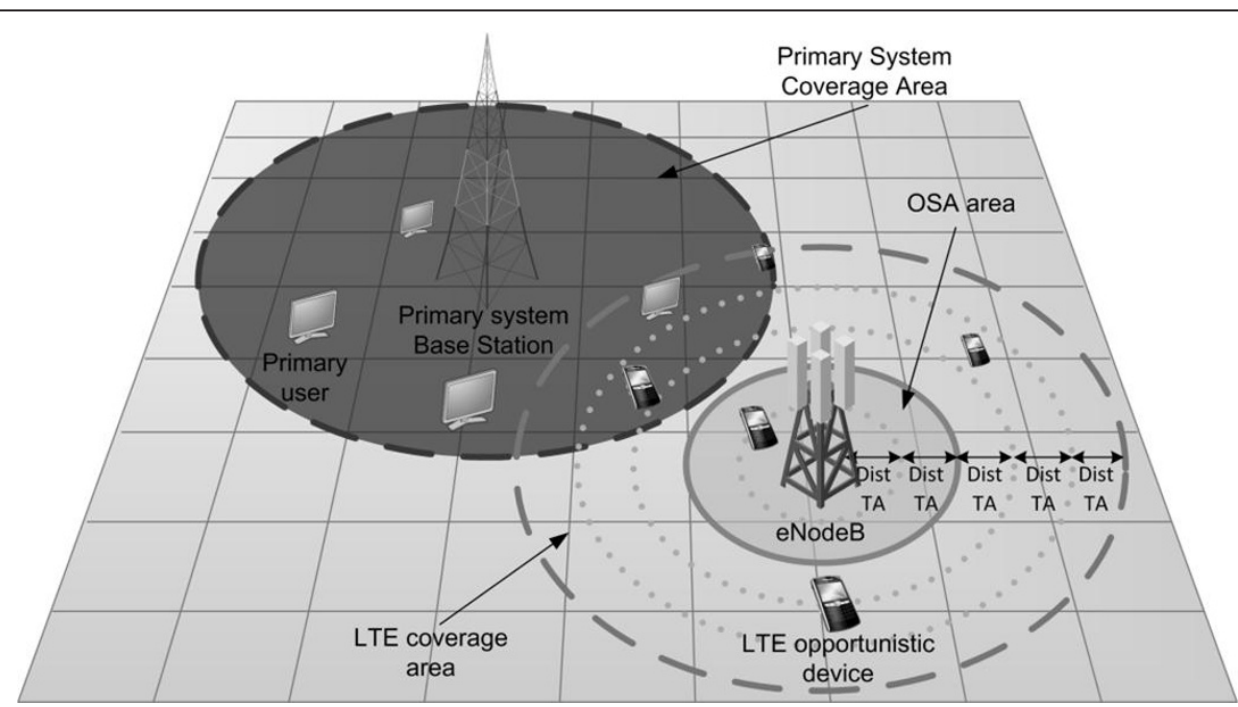

Figure 6 Example of opportunistic LTE scenario. Example of a possible scenario where a LTE system opportunistically operates in another licensed frequency band without interfering. 
Each eNodeB has a maximum transmit power to be distributed among all available RBs. This means that opportunistic resources that are going to be allocated must be taken into account in the distribution of power. Our proposed system will query the Geo-DB to discover which licensed resources the LTE system can use in an opportunistic way and, then, the scheduler will eventually decide when to use them. Once the system knows the total amount of resources to be allocated in a given eNodeB, it also knows the maximum power transmission per $R B$, just dividing the total transmit power available in the eNodeB by the number of RBs to allocate. For the opportunistic RBs, power restrictions may apply if stated in the Geo-DB and the scheduler must adjust the transmission power according to the maximum distance the LTE signal must not exceed. The procedure to adjust the transmission implies reducing the maximum transmission power considering the difference in propagation losses between the maximum coverage distance of the LTE cell-given by the $95 \%$-tile of the distance of located users, extracted from off-line statistics-and the maximum distance where the opportunistic resource can be used.

In order to investigate the advantages of such PC strategy, it will be compared with a non-PC procedure in Section 4. As its name suggests, non-PC implies not adjusting the transmit power of the opportunistic signal and hence the eNodeB transmits with the maximum power available whenever the cooperative decision-making mechanism concludes the resource is free.

The reduction of the transmission power must be followed by a correction of the CQI reported by the UE in order to use the right modulation and coding scheme (MCS) for that power. Note that, according to LTE specifications, decreasing the transmission power by $2 \mathrm{~dB}$ corresponds to decreasing the CQI by 1 [29].

Once resources (i.e., the RBs) have been assigned to opportunistic users, user mobility adds an increased complexity due to the increase of system dynamics. Moreover, the localization procedure may report user position with some inaccuracy. Both aspects may lead opportunistic users to interfere with the primary activity. A survey about the impact of the location precision error in the system performance is detailed in Section 4.

\subsection{Access procedure}

As stated in the introduction, in LTE-A the amount of resources can be increased by aggregating continuous or discontinuous portions of spectrum-referred to as component carriers $(\mathrm{CCs})$-in order to provide higher data rates. In the context of $C R$, the $C A$ concept can be extended and additional portions of spectrum can be used on an opportunistic and non-interfering basis by adding the detected spectrum holes or white spaces. This is the main concept on which OSA relies, providing extended capabilities and improved flexibility in the aggregation of spectrum resources and enhancing both data rate and spectrum efficiency.

On each CC, it is necessary to adjust the opportunistic transmission parameters (e.g., transmit power, modulation and coding schemes, etc.) to the available spectrum holes. As a result, separate Hybrid Automatic Repeat reQuest (HARQ) processing and its associated control signaling are required for each CC. In this situation, the proper design of the control signaling channel is crucial. In general, according to 3 GPP internal discussions there are three possible implementations of the control channel in CA [38]: (a) each CC can have its own coded control channel and minor modifications of the control structure in LTE systems are required (Figure 7a), (b) the control channels of different CCs can be jointly coded and transmitted in a dedicated CC (Figure 7b), and (c) multiple control channels for different CCs are jointly coded and then transmitted over the entire frequency band formed by the licensed LTE band and the

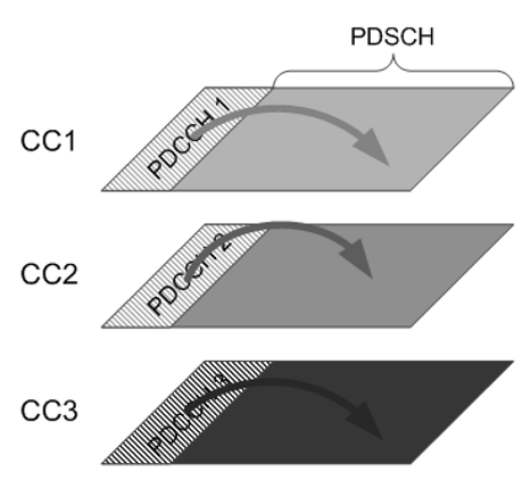

a)

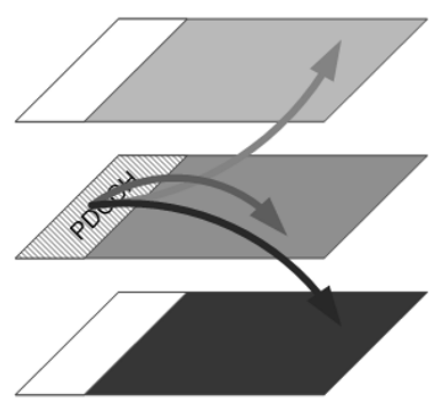

b)

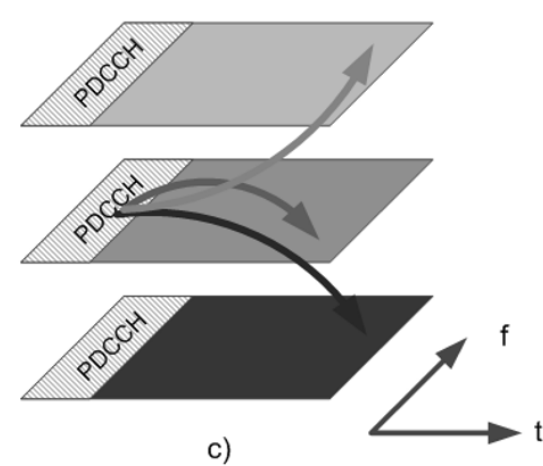

c)

Figure 7 PDCCH designs for LTE-A when CA is enabled 
CC added (Figure 7c). Approaches (a) and (c) are incompatible with OSA since prior LTE signaling transmission is required in each $\mathrm{CC}$ in the licensed band before knowing if that specific $\mathrm{CC}$ is idle, increasing collisions and interference with the licensed activity. Therefore, the proposed system requires the implementation of the control signaling scheme (b), where the signaling from all the added CC is carried in the licensed LTE control channel. Opportunistic users will read their signaling information in the licensed LTE band and then, according to that information, transmit or receive data in the opportunistic CCs. This procedure must be performed periodically in order to release the opportunistic resources if the primary activity returns. LTE handsets can carry out this operation when all the CCs are contiguous, including the licensed LTE band [2]. However, in case that CCs are discontinuous, and assuming that mobile devices only have a single radio interface, once the opportunistic user reads the control channel and finds out its allocated resources the handset has to tune its working frequency to the allocated $\mathrm{CC}$, synchronize to the LTE system to start data transmission or reception and, after a specific time interval, re-tune the radio to the licensed LTE band and read the control channel again. This situation is impractical in the ambit of OSA since the required time between re-tunes must be very short in order to provide updated information of the opportunistic resources availability and, even if the handset is able to perform fast re-tuning, there is no useful time left to exploit those frequencies. A feasible solution to this problem is to implement a semi-persistent scheduling [39] where the signaling check periodicity is extended (up to several seconds) without taking into account the licensed channel state. The longer the time the opportunistic user does not consult the control channel, the higher the probability that licensed and unlicensed activities collide. Thus, a signaling consulting period and collision probability trade-off must be met.

Noteworthy, the use of OFDM-based opportunistic systems comes at a reduced cost since a particular set of subcarriers may be fed by zeroes in the corresponding transmitter's Inverse Fast Fourier Transform (IFFT) input to prevent interference with the primary system. At the receiver, the FFT operation implemented to recover the transmitted data will still be valid for the OSA operation mode, with no extra cost. In [40], an efficient implementation of a Non-Contiguous OFDMA (NC-OFDMA) transceiver is presented for $\mathrm{CR}$ applications.

\section{Results}

\subsection{Simulation scenario and parameter setup}

We consider an illustrative scenario where a single LTE$\mathrm{A}$ eNodeB and a licensed base station coexist. The primary system exploits the $2.3 \mathrm{GHz}$ band while the LTE-
A system carrier frequency is $2 \mathrm{GHz}$. The coverage areas of both systems partially overlap as represented by Figure 6 . The overlapping degree can be varied in order to study to what extent OSA provides substantial improvement of capacity depending on the distance between the primary and the secondary system. Opportunistic LTE-A users were randomly spread throughout the LTE-A system coverage area. Primary system transmissions were randomly generated following the semiMarkov ON-OFF Pareto-distribution [41]. Its probability distribution function $f(x)$ is defined as follows:

$$
f(x)=k \frac{x_{m}^{k}}{x^{k+1}}, x \geq x_{m}
$$

where $k$ is referred to as the shape parameter and $x_{m}$ is the scale parameter of the Pareto distribution. Note that different combinations of these two parameters result in different primary activity periodicities, $T_{\mathrm{PA}}$, i.e., different average ON-OFF intervals in the primary system. In the simulator, the primary activity is modeled by the primary activity factor $\left(F_{\mathrm{PA}}\right)$, which normalizes the $T_{\mathrm{PA}}$. Table 4 shows the most relevant simulation parameters used in this study.

In this study two different metrics are used: the maximum throughput served by the eNodeB with full-buffer UEs and the collision ratio that models the probability that the LTE-A activity interferes with the licensed system. Collision ratio is calculated as the ratio of the number of times OSA is performed when the licensee is active to the number of times a secondary UE in the coverage area of both licensed and non-licensed system uses opportunistically a licensed RB. In this article, the target collision ratio is set to be below $10 \%$.

\subsection{Sensing calibration}

Before obtaining performance results from the implementation of the suggested opportunistic tools in a LTE-A system, first it is necessary to optimize the cooperative spectrum sensing mechanism in order to provide the best OSA performance. This optimization requires setting the most suitable decision threshold for the softdecision cooperative spectrum sensing algorithm, described in Section 3.1, and, according to this threshold, setting an appropriate resource sensing periodicity for UEs, i.e., MGRP.

In Figure 8, it is shown the performance of the opportunistic LTE-A system in terms of cell throughput and collision ratio for a decision threshold $\gamma$, ranging from -1 to 1 . This figure compares the performance of the soft-based cooperative decision procedure assuming linear weights (Equation (3)), but also the quadratic (Equation (4)) and the square root version (Equation (5)). In this analysis, resource sensing periodicity was set to 40 $\mathrm{ms}$. Lower decision thresholds imply to be less confident 
Table 4 Simulation parameters

\begin{tabular}{cc}
\hline Parameter & Value \\
\hline Cell layout & 1 omni-directional cell/1 primary transmitter \\
\# users & 100 \\
LTE-A carrier frequency/bandwidth & $2 \mathrm{GHz} / 5 \mathrm{MHz}(25 \mathrm{RBs})$ \\
Primary carrier frequency/bandwidth & $2.3 \mathrm{GHz} / 5 \mathrm{MHz}(25 \mathrm{RBs})$ \\
Scheduling & Round Robin (LTE-A band)/MaxCIR [43] (Opportunistic band) \\
Propagation model & Urban Macro (UMa) [44] \\
Shadowing model & Log-normal, $\sigma=4 \mathrm{~dB}$ \\
$k$ & 2 \\
$x_{m}$ & 60 \\
Average primary system ON-OFF interval $\left(T_{\mathrm{PA}}\right)$ & $50 \mathrm{~s}$ \\
Max. number of scheduled users & 10 \\
Mobility & Static users \\
FA/MD Probabilities & $0.028 / 0.01$ (target) \\
Time-bandwidth product $(m)$ & 930 \\
Received primary SNR $\left(\bar{\gamma}_{p}\right)$ & $8 \mathrm{~dB}$ \\
Simulation time & $1000 \mathrm{~s}$ \\
\hline
\end{tabular}

on the channel vacuity before using this resources, what increases collision probability. Conversely, higher decision thresholds entail less collisions but also a significant reduction in capacity because the OSA capability is wasted. As it can be seen, a good choice providing both maximum throughput along with low collision probability is to take $\gamma$ equal to zero. Accordingly, this value will be used in the remainder. It can also be appreciated that similar results are achieved with linear and quadratic weight formulas at $\gamma=0$. So, it is suggested to use the linear version provided its lower complexity.

Once the optimum decision threshold is set, it is necessary to check the benefits of soft-decisions as compared to hard decisions. For this purpose, Figure 9 depicts the experienced system performance (in terms of cell throughput and collision ratio) for different

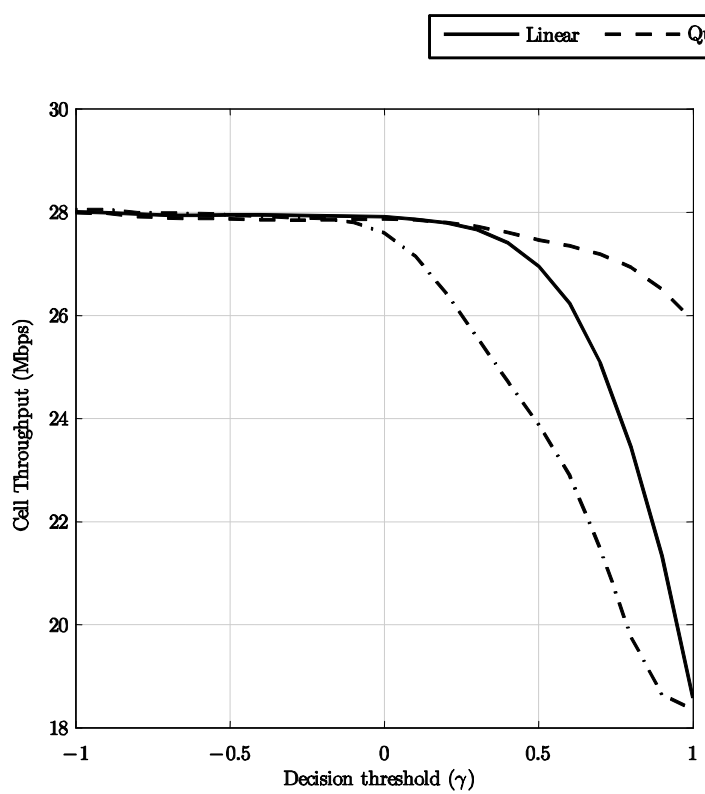

(a)

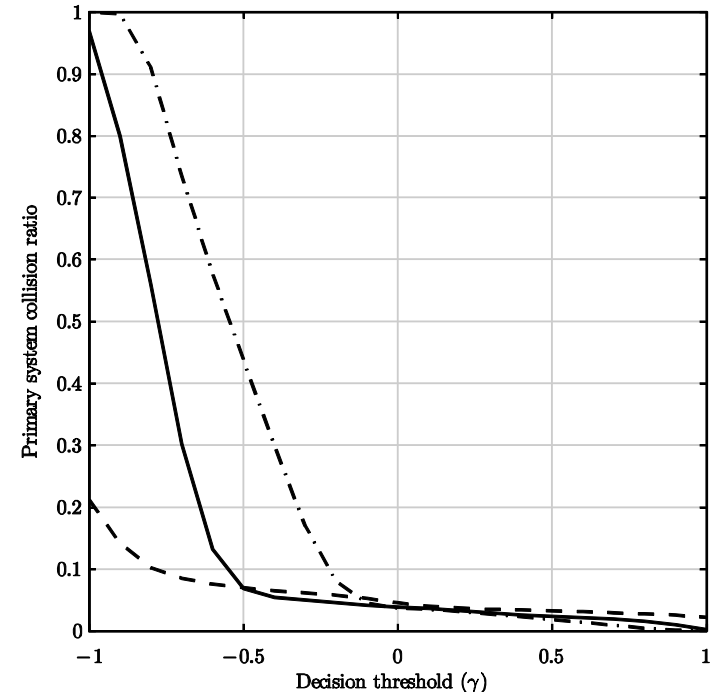

(b)

Figure 8 System performance against decision thresholds. System performance in terms of (a) cell throughput and (b) collision ratio for increasing decision thresholds when the linear, quadratic and square root versions are used. 


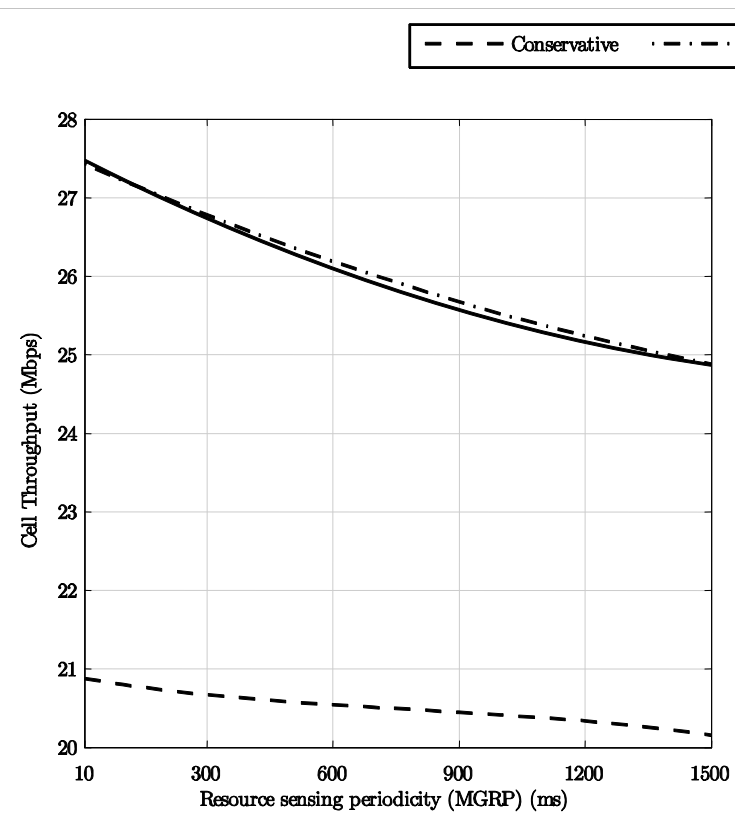

(a)

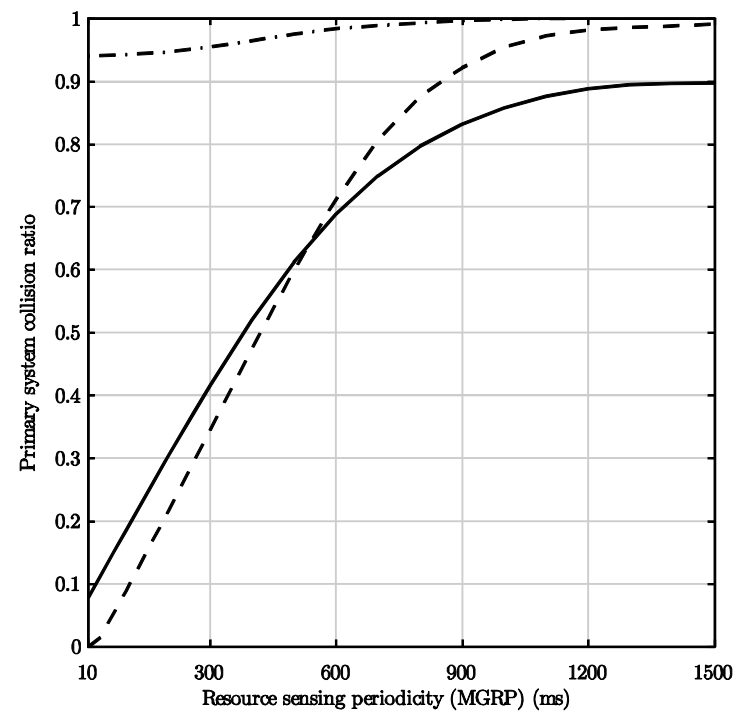

(b)

Figure 9 System performance against decision mechanisms. Comparison of cell throughput (a) and collision ratio (b) among the conservative (dashed), aggressive (dotted-dashed) and soft-cooperative (solid) decision-making mechanisms considered for different sensing periodicities (MGRP).

sensing periodicities, i.e., different values of MGRP, with the three decision-making algorithms detailed in Section 3.1. Solid curves correspond to the soft-decision algorithm proposed in this article, the dashed curve is for the conservative strategy and, finally, the dash-dotted line is for the aggressive strategy. Figure 9a shows that the proposed soft-decision algorithm provides similar cell throughput as compared to the aggressive strategy. This is mainly due to the fact that UE sensing intervals, i.e., the 6 ms-long MGL (see Section 2.1) when the UE performs the spectrum sensing task, are unsynchronized and some users can consume resources while others are sensing. However, longer sensing periods increase the channel uncertainty and make opportunistic users collide with the primary system. It can be also seen that increasing the sensing periodicity, which reduces the number of spectrum queries for a given time, does not enhance data throughput as it may be expected. Moreover, if the sensing periodicity is too high, channel state information is outdated and the number of collisions rises, see Figure 9b, reducing the throughput. In the remainder, the sensing period, or MGRP, will be assumed equal to $10 \mathrm{~ms}$ since this value implies the highest cell throughput and lowest possible collision ratio according to the standard [28], which specifies that MGRP is configurable in multiples of the frame lengthi.e., $10 \mathrm{~ms}-($ see Figure 1).

\subsection{Cooperative algorithm evaluation}

Another critical aspect in OSA techniques relying on Geo-DBs is the accuracy of the adopted UE location method. If a given UE is incorrectly positioned in an area where OSA is allowed, but its real location is in a forbidden OSA area, occupied opportunistic resources will be allocated to that user, therefore interfering with the licensed system. In addition, more location-error problems may appear when the power of the opportunistic activity in the licensed band has to be limited due to the detection of primary activity. In case the maximum range of the opportunistic signal is over-dimensioned, the number of collisions will increase. As a result, the performance provided by the PC mechanism (see Section 3.3) and, hence, the performance of OSA, may be compromised. Aiming at studying the impact of the precision error of the UE localization method, a precision error modeled as a Gaussian distribution has been introduced. In this way, Figure 10 shows the difference between either implementing PC (solid curve) or not (dashed lines) for different location errors. As it can be expected, the lack of a mechanism that controls the power of the opportunistic system causes harmful interferences with the licensee, increasing the collision ratio, and limiting the potential throughput. On the other hand, simulation results show that the average cell throughput is slightly affected by the UE positioning 


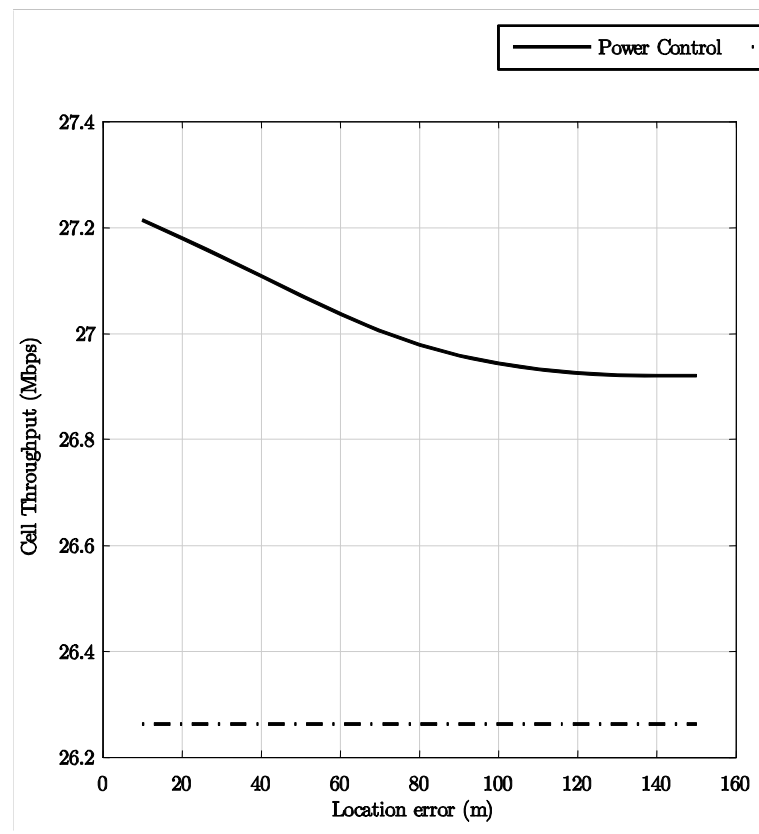

(a)

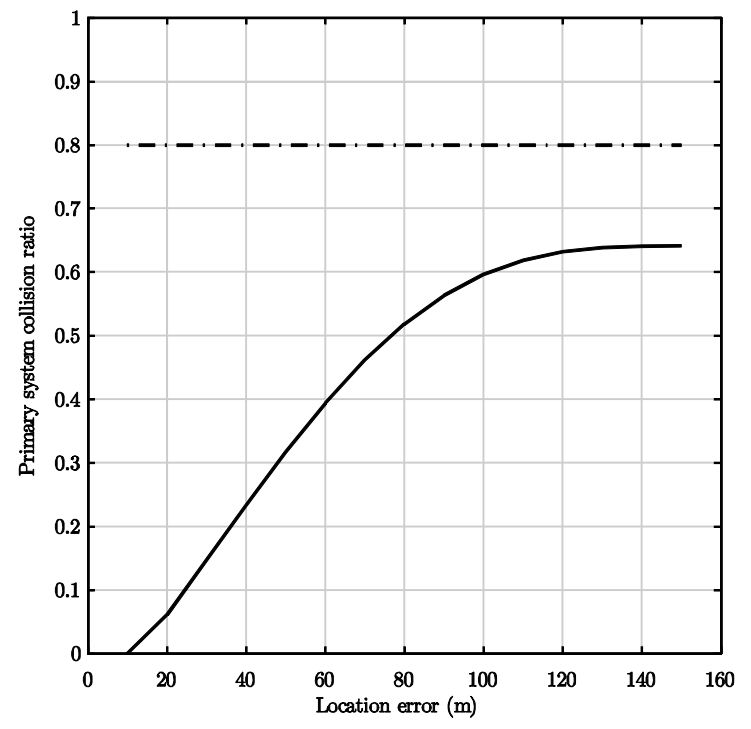

(b)

Figure 10 Impact of location precision errors. (a) Cell throughput and (b) primary collision ratio for different location precision errors, with and without power control.

precision. Positioning precision mainly affects the collision probability in the LTE-A system for the most distant users from the eNodeB, consequently having their transmission power reduced to the minimum. In Figure $10 \mathrm{~b}$ it can be seen that the maximum location error allowed in order to have a collision ratio lower than $10 \%$ is $30 \mathrm{~m}$. In any case, there is a significant difference between implementing the $\mathrm{PC}$ and the Non-PC strategies for the allocation of resources in OSA. In the NonPC case, the eNodeB transmits with the maximum power per RB, without power control, when it is cooperatively decided that there is no primary activity, increasing the number of collisions. As the scheduler used for the opportunistic resources tries to maximize the throughput (MaxCIR), the opportunistic resources will be normally scheduled for users close to the eNo$\mathrm{deB}$ and the collision probability in the LTE-A system will be small. Despite this, in the event of scheduling users far from the eNodeB, as they are closer to the primary system, their MCS will be more robust hence reducing the amount of transmitted data. Despite this reduction of transmitted data, the effect over the global throughput will be minor.

\subsection{Overlapping and system activity impact}

In a real scenario, base stations are spread so as to cover the whole service area. For this reason, the distance between eNodeBs and the primary system may change from one site to other. This may result in a lower or higher overlapping degree between the primary and the opportunistic systems, if any overlapping at all. Therefore, it is necessary to evaluate the performance of the opportunistic tools described in this study for different overlapping situations. The overlapping factor $(O F)$ is defined as the percentage of users under the LTE-A coverage area that are also able to detect the primary system activity. In addition, the primary system activity in a certain period of time depends on the primary system and its specific type of traffic. This section addresses the dependence of the proposed OSA upon these two parameters.

Figure 11 depicts the cell throughput of LTE-A against increasing $O F$ for different primary activity factors (see $F_{\mathrm{PA}}$ definition in Section 4.1). Results show that with more activity in the primary system fewer resources are available for OSA and, thus, the achieved bit rate is lower. The overlapping factor also impacts on the experienced performance, especially for primary activity periodicities greater than $40 \%$ of the simulation time. However, the number of collisions is not affected by the overlapping area but rather by the primary system activity, as shown in Figure 12. Indeed, collision ratio increases when primary activity time decreases. The reason for this is that collisions are due to the sudden changes in the primary activity state. 


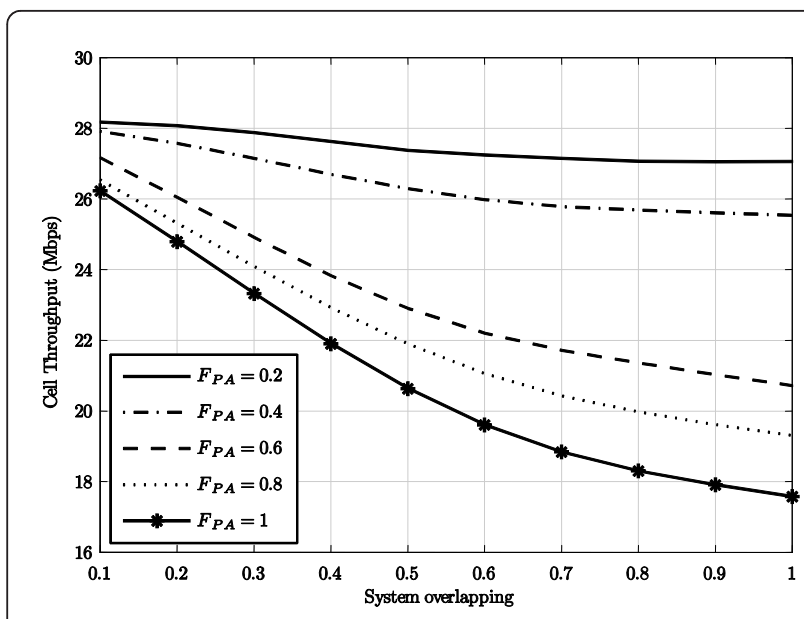

Figure 11 Cell throughput against system overlapping and primary system activity factor. Experienced cell throughput for different system overlapping $(O F)$ and primary system activity factor $\left(F_{P A}\right)$ values.

\section{Conclusions}

This study has proposed a set of tools and procedures to include OSA in an LTE-A system. The implementation of this opportunistic access in LTE-A certainly enhances the overall system performance by intelligently aggregating otherwise unutilized spectrum. However, as discussed throughout the article, the opportunistic mechanisms must be set-up carefully taking into account the implementation feasibility offered by the adopted standards. First, sensing periods must be as small as possible in order to increase sensing accuracy.

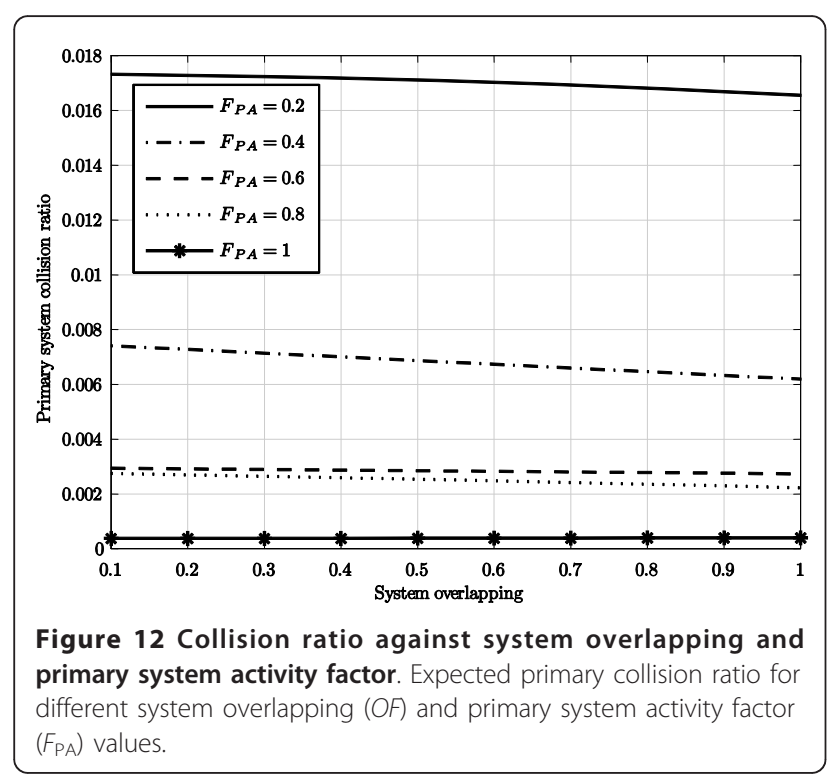

Table 5 List of notations

\begin{tabular}{|c|c|}
\hline Acronym & Definition \\
\hline $3 \mathrm{GPP}$ & 3rd generation partnership project \\
\hline A-GNSS & Assisted-Global navigation Satellite system \\
\hline AoA & Angle of arrival \\
\hline CA & Carrier aggregation \\
\hline CC & Component carrier \\
\hline$C R$ & Cognitive radio \\
\hline CRM & Cognitive resource manager \\
\hline Dist TA & Timing advance resolution distance \\
\hline DTV & Digital television \\
\hline $\mathrm{E}-\mathrm{CID}$ & Enhance cell ID \\
\hline $\mathrm{ECGl}$ & Evolved cell global identifier \\
\hline E-SMLC & Enhanced service mobile location center \\
\hline FA & False alarm \\
\hline Geo-DB & Geo-located database \\
\hline IMT-A & International mobile telecommunications-advanced \\
\hline LCS-AP & Location services application protocol \\
\hline LPP & LTE positioning protocol \\
\hline LPPa & LTE position protocol annex \\
\hline LTE & Long term evolution \\
\hline LTE-A & Long term evolution-advanced \\
\hline MaxCIR & Maximum carrier-to-interference ratio \\
\hline MD & Missed detection \\
\hline MGL & Measurement gap length \\
\hline MGRP & Measurement gap repetition period \\
\hline $\mathrm{MlH}$ & Media independent handover \\
\hline MME & Mobility management entity \\
\hline NC-OFDMA & Non-contiguous OFDMA \\
\hline N.A. & Not applicable \\
\hline OFDM & Orthogonal frequency division multiplexing \\
\hline OFDMA & Orthogonal frequency-division multiple access \\
\hline OTDoA & Observed time difference of arrival \\
\hline OSA & Opportunistic spectrum access \\
\hline PC & Power control \\
\hline PRS & Positioning reference signals \\
\hline $\mathrm{RB}$ & Resource block \\
\hline $\mathrm{ROC}$ & Receiver operating characteristic \\
\hline RSSI & Received signal strength indicator \\
\hline RSRQ & Reference signal received quality \\
\hline$R_{x}$ & Receiver \\
\hline SNR & Signal-to-noise ratio \\
\hline TA & Timing advance \\
\hline TETRA & Terrestrial trunked radio \\
\hline$T_{s}$ & Sensing time \\
\hline$T_{\text {samp }}$ & Sampling period \\
\hline$T_{p}$ & Sensing periodicity \\
\hline$T_{x}$ & Transmitter \\
\hline UE & User equipment \\
\hline UMa & Urban Macro \\
\hline
\end{tabular}


Only by using cooperative decision-making mechanism these sensing periods can be increased. Moreover, several mechanisms for cooperative decision have been compared. It has been proven that the cooperative soft decision-making algorithm proposed in this article provides better performance than other hard decision mechanisms found in the literature. Similar rates to the aggressive strategy are achieved with the soft criterion, but with an evident reduction of the number of collisions with the licensed system, even lower than those achieved with the conservative strategy. Therefore, the suggested soft-decision algorithm takes advantage of the positive aspects of the two hard-decision criteria if an appropriate decision threshold is chosen. Finally, the UE location mechanism must be accurate enough in order to avoid interferences with the primary system. Specific figures have been provided for this level of accuracy.

The OSA method described in this article clearly enhances the LTE-A system performance. In addition, the proposed system is a low-cost solution because there is no need for a substantial modification of the LTE-A system architecture. On the contrary, only a new functional entity must be added to the system, together with a set of new application layer services. This new entity, the CRM, should be preferably collocated with the MME entity, so as to reduce the signaling overhead.

\section{Appendix 1: List of notations}

Table 5 lists the most frequently used abbreviations in this article.

\section{Endnotes}

"We will interchangeably use the terms "channel", as in a frequency channel, and "band" when referring to a portion of spectrum potentially available for OSA. ${ }^{\mathrm{b}} \mathrm{An} \mathrm{RB}$, using 3GPP terminology, is the minimum portion of spectrum that can be assigned to a particular user $(180$ $\mathrm{kHz}$ ). It may be used in the same context as with the term "channel". 'Note that RSSI measurements are made calculating the total signal power received in the antenna port after applying a specific shape filter. However, due to backward compatibility, a GSM-specific shape filter of $200 \mathrm{kHz}$ is available at the LTE equipments that can be used to measure the signal level within an RB [42].

\footnotetext{
Acknowledgements

The authors would like to thank the funding received from the Ministerio de Ciencia e Innovación within the Project number TEC2011-27723-C02-02 and from the Ministerio de Industria, Turismo y Comercio TSI-020100-2011-266 funds. This article had been written in the framework of the CELTIC project CP08-001 COMMUNE. Study by X. Gelabert is funded by the BP-DGR 2010 scholarship (ref. 00192). The authors would like to acknowledge the contributions of their colleagues.
}

\section{Author details}

${ }^{1}$ Universitat Politècnica de València, iTEAM Research Institute, Camino de Vera S/N, 46022 València, Spain ${ }^{2}$ Centre for Telecommunications Research (CTR), King's College London (KCL), Strand, London WC2R 2LS, UK

\section{Competing interests}

The authors declare that they have no competing interests.

Received: 13 September 2011 Accepted: 9 March 2012 Published: 9 March 2012

\section{References}

1. D Martín-Sacristán, JF Monserrat, J Cabrejas-Peñuelas, D Calabuig, S Garrigas, N Cardona, On the way towards fourth-generation mobile: 3GPP LTE and LTE-Advanced. EURASIP J Wirel Commun Netw. 2009, 1-10 (2009)

2. R Ratasuk, D Tolli, A Ghosh, Carrier aggregation in LTE-Advanced, in IEEE 71st Vehicular Technology Conference (VTC 2010-Spring), Taipei, pp. 1-5 (2010)

3. H Wang, C Rosa, K Pedersen, Performance of uplink carrier aggregation in LTE-advanced systems, in IEEE 72nd Vehicular Technology Conference Fall (VTC 2010-Fall), Ottawa, pp. 1-5 (2010)

4. R Tandra, A Sahai, S Mishra, What is a spectrum hole and what does it take to recognize one?. Proc IEEE. 97(5), 824-848 (2009)

5. IJ Mitola, JGQ Maguire, Cognitive radio: making software radios more personal. IEEE Personal Commun. 6(4), 13-18 (1999). doi:10.1109/98.788210

6. S Haykin, Cognitive radio: brain-empowered wireless communications. IEEE J Sel Areas Commun. 23(2), 201-220 (2005)

7. IEEE 802.22 Working Group on Wireless Regional Area Networks http:// www.ieee802.org/22/

8. ITU-R BT1368: Planning criteria for digital terrestrial television services in the VHF/UHF bands

9. ITU-R BT1786: Criterion to assess the impact of interference to the terrestrial broadcasting service (BS)

10. S Kawade, M Nekovee, Cognitive radio-based urban wireless broadband in unused TV bands, in 20th International Radioelektronika Conference, Brno, pp. 1-4 (2010)

11. B Modlic, G Sisul, M Cvitkovic, Digital dividend-Opportunities for new mobile services, in International Symposium ELMAR 2009 (ELMAR'09), Zadar, pp. 1-8 (2009)

12. X Zhao, Z Guo, Q Guo, A cognitive based spectrum sharing scheme for LTE advanced systems, in International Congress on Ultra Modern Telecommunications and Control Systems and Workshops (ICUMT), Moscow, pp. 965-969 (2010)

13. S Hussain, X Fernando, Spectrum sensing in cognitive radio networks: Upto-date techniques and future challenges, in IEEE Toronto International Conference on Science and Technology for Humanity (TIC-STH), Toronto, pp. 736-741 (2009)

14. $Y X u, Y$ Sun, $Y$ Li, Y Zhao, H Zou, Joint sensing period and transmission time optimization for energy-constrained cognitive radios. EURASIP J Wirel Commun Netw. 2010, 1-16 (2010)

15. T Yucek, H Arslan, A survey of spectrum sensing algorithms for cognitive radio applications. IEEE Commun Surv Tutor. 11, 116-130 (2009)

16. D Cabric, S Mishra, R Brodersen, Implementation issues in spectrum sensing for cognitive radios, in Conference Record of the Thirty-Eighth Asilomar Conference on Signals, Systems and Computers, vol. 1. (Pacific Grove, 2004), pp. 772-776

17. Y Zeng, YC Liang, A Hoang, E Peh, Reliability of spectrum sensing under noise and interference uncertainty, in IEEE International Conference on Communications Workshops, 2009. ICC Workshops, Dresden, pp. 1-5 (2009)

18. L Bixio, M Ottonello, M Raffetto, CS Regazzoni, Comparison among cognitive radio architectures for spectrum sensing. EURASIP J Wirel Commun Netw. 2011, 1-18 (2011)

19. M Mustonen, M Matinmikko, A Mammela, Cooperative spectrum sensing using quantized soft decision combining, in 4th International Conference on Cognitive Radio Oriented Wireless Networks and Communications, 2009 (CROWNCOM'09), Hannover, pp. 1-5 (2009)

20. L Xiao, K Liu, L Ma, A weighted cooperative spectrum sensing in cognitive radio networks, in International Conference on Information Networking and Automation (ICINA), vol. 2. Kunming, pp. 45-48 (2010) 
21. Q Pan, Y Chang, R Zheng, X Zhang, Y Wang, D Yang, Solution of information exchange for cooperative sensing in cognitive radios, in IEEE Wireless Communications and Networking Conference, 2009 (WCNC'2009), Budapest, pp. 1-4 (2009)

22. A Masri, CF Chiasserini, A Perotti, Control information exchange through UWB in cognitive radio networks, in 5th IEEE International Symposium on Wireless Pervasive Computing (ISWPC), Modena, pp. 110-115 (2010)

23. $\mathrm{H}$ Celebi, $\mathrm{H}$ Arslan, Utilization of location information in cognitive wireless networks. IEEE Wirel Commun. 14(4), 6-13 (2007)

24. FCC: Notice of Proposed Rulemaking, in the Matter of Unlicensed Operation in the TV Broadcast Bands (ET Docket no. 04-186) and Additional Spectrum for Unlicensed

25. MJ Marcus, P Kolodzy, A Lippman, Reclaiming the vast wasteland: why unlicensed use of the white space in the TV bands will not cause interference to DTV viewers. New America Foundation: wireless future program, tech rep (2005)

26. H Nam, M Ghorbel, M Alouini, in Proc of the Fifth International Conference on Cognitive Radio Oriented Wireless Networks Communications (CROWNCOM), Cannes, pp. 1-5 (2010)

27. IEEE Std 80221-2008: IEEE Standard for Local and Metropolitan Area Networks-Part 21: Media Independent Handover (2009)

28. 3GPP TS 36133: Evolved Universal Terrestrial Radio Access (E-UTRA); Requirements for support of radio resource management

29. S Sesia, M Baker, I Toufik, LTE, the UMTS long term evolution: from theory to practice, (Wiley, New Haven, 2009)

30. FF Digham, MS Alouini, MK Simon, On the energy detection of unknown signals over fading channels, in IEEE International Conference on Communications, 2003 (ICC'03), vol. 5. Anchorage, pp. 3575-3579 (2003)

31. A Ghasemi, ES Sousa, Collaborative spectrum sensing for opportunistic access in fading environments, in First IEEE International Symposium on New Frontiers in Dynamic Spectrum Access Networks (DySPAN), Baltimore, pp. 131-136 (2005)

32. X Gelabert, IF Akyildiz, O Sallent, R Agustí, Operating point selection for primary and secondary users in cognitive radio networks. Comput Netw. 53(8), 1158-1170 (2009). doi:10.1016/j.comnet.2009.02.009

33. K Taniuchi, Y Ohba, V Fajardo, S Das, M Tauil, YH Cheng, A Dutta, D Baker, M Yajnik, D Famolari, IEEE 802.21: media independent handover: features, applicability, and realization. IEEE Commun Mag. 47, 112-120 (2009)

34. 3GPP TS 36305: Evolved Universal Terrestrial Radio Access Network (EUTRAN); Stage 2 functional specification of User Equipment (UE) positioning in E-UTRAN

35. 3GPP TS 36355: Evolved Universal Terrestrial Radio Access; LTE Positioning Protocol (LPP)

36. 3GPP TS 36455: Evolved Universal Terrestrial Radio Access; LTE Positioning Protocol A (LPPa)

37. W Ren, Q Zhao, A Swami, Power control in cognitive radio networks: how to cross a multi-lane highway. IEEE J Sel Areas Commun. 27(7), 1283-1296 (2008)

38. 3GPP R1-084424: Control Channel Design Issues for Carrier Aggregation in LTE-A

39. J Dajie, W Haiming, E Malkamaki, E Tuomaala, Principle and performance of semi-persistent scheduling for VoIP in LTE system, in International Conference on Wireless Communications, Networking and Mobile Computing, 2007 (WiCom 2007), Shanghai, pp. 2861-2864 (2007)

40. R Rajbanshi, AM Wyglinski, GJ Minden, An efficient implementation of NCOFDM transceivers for cognitive radios, in Proc of 1st Conf on Cognitive Radio Oriented Wireless Networks and Commun, Mykonos, pp. 1-5 (2006)

41. M Wellens, J Riihijarvi, P Mahonen, Modeling primary system activity in dynamic spectrum access networks by aggregated ON/OFF-processes, in 6th Annual IEEE Communications Society Conference on Sensor, Mesh and Ad Hoc Communications and Networks Workshops, 2009. SECON Workshops'09, Rome, pp. 1-6 (2009)

42. 3GPP TS 36214: Physical layer; Measurements

43. Y Ofuji, A Morimoto, S Abeta, M Sawahashi, Comparison of packet scheduling algorithms focusing on user throughput in high speed downlink packet access, in 13th IEEE International Symposium on Personal, Indoor and Mobile Radio Communications, vol. 3. Lis-boa, pp. 1462-1466 (2002)

44. ITU-R ITU M2135: Guidelines for evaluation of radio interface technologies for IMT-Advanced (2008) doi:10.1186/1687-1499-2012-99

Cite this article as: Osa et al.: Implementing opportunistic spectrum access in LTE-advanced. EURASIP Journal on Wireless Communications and Networking 2012 2012:99.

\section{Submit your manuscript to a SpringerOpen ${ }^{\mathcal{O}}$ journal and benefit from:}

- Convenient online submission

- Rigorous peer review

- Immediate publication on acceptance

- Open access: articles freely available online

- High visibility within the field

- Retaining the copyright to your article

Submit your next manuscript at $>$ springeropen.com 